\title{
Numerical Simulation of Mining Machine Cutter's Thermal Spraying Process
}

\author{
Hanwu Liu \\ Sch. of Mechanical and Electrical Engineering \\ North China Institute of Science and Technology \\ Sanhe, Hebei, China \\ e-mail: hanwu-liu@ sohu.com
}

Chao Cao

Sch. of Mechanical and Electrical Engineering

North China Institute of Science and Technology

Sanhe, Hebei, China

e-mail: 352238921@qq.com

\author{
Zhiping Zhang \\ Sch. of Humanities and Social Sciences \\ North China Institute of Science and Technology \\ Sanhe, Hebei, China \\ e-mail: zhipingzhanghk@sohu.com
}

\begin{abstract}
In this paper, Finite Element technique is adopted to take the numerical simulation of mining machine cutter's thermal spraying process. First, a FE model of flat particle is built to analyze the temperature distribution during the process of flat particle's heat exchange with base metal. Then, in order to simulate the temperature distribution and stress field variation with time as the molten drops deposit and solidify on base metal, the indirect coupling method, which loads previous results of temperature field on the model, is used to calculate the stress distribution in flat particle. Based on LS-DYNA module, the stress and strain distributions of spraying particle in the process of collision, deposition and deformation are analyzed, and the contour maps of stress or strain and energy curves in the collision process are obtained. Accordingly, the effect of preheating temperature of base metal on stress distributions is analyzed further. The results of this study not only provide theoretical support for the deposition and forming technique of plasma drop, but also offer a new research tool for studying spraying temperature, velocity and the optimization of coating interface.
\end{abstract}

Keywords-plasma spray; molten drop; temperature distribution; residual stress; coupling analysis

\section{INTRODUCTION}

At work, a mining machine cutter bears high compressive stress, shear stress and impact loads, and with the increasing working hours, the temperature of cutter will increase rapidly, all of which can easily cause various forms of failure such as premature wear of cutter, the loss and fragmentation of cutter head. In order to improve the life of cutter and lower the production difficulty, plasma spraying and other advanced treatment techniques can be adopted to harden the surface of cutter head.

Thermal spraying technology is widely used in aviation, aerospace, machinery, bio-engineering, nuclear engineering and many other fields for the manufacture of coating with high temperature resistance, wear resistance, corrosion resistance and other features, and it can also be used for the production of metal or ceramic parts and molds without shape reprocessing. But the process of thermal spraying is so complex with physical and chemical changes involved in comprehensive knowledge of heat, phase transition, mechanics and dynamics that there are still no complete theories or mathematical methods to describe this procedure accurately. With the rapid development of computer technology, it is possible to describe the thermal spraying process by using computer simulation technique. As FEM can easily handle the nonlinear properties of materials, particularly suitable to the case of fields with multi-media and junctions with complex shape, therefore, it can be used to simulate the variation law of various mechanical parameters of materials during the thermal spraying process.

In this paper, ANSYS software is used to build a mathematical model of molten drops depositing and solidifying on the surface of base metal, and simulate the temperature and residual stress distributions after the drops deposit and solidify on base and the deformation process of drops during the plasma spraying process. In order to realize efficient, stable plasma spraying processing of cutter head's surface with little or no pores and defects, the temperature field and stress field are analyzed by using thermal stress indirect coupling analysis method, and the deformation process is simulated by using ANSYS / LS-DYNA module.

II. THE SIMULATIONS OF TEMPERATURE FIELD, STRESS FIELD AND OF THERMAL SPRAYING PROCESS AFTER MOLTEN DROPS DEPOSIT ON BASE METAL

\section{A. Mathematical Model of Molten Drop Depositing on Base Metal.}

During the plasma spraying process, Ni metal drops deposit on the infinite surface of carbon steel base at a certain speed. After diffusing with deformation and changing heat with base, the drops solidify into disc-shaped solid particles. The longitudinal shape and size of solid particle is shown in Fig.1. It is assumed that the initial melting 
temperature of particle is $1454^{\circ} \mathrm{C}$, and the particles cool to room temperature $25^{\circ} \mathrm{C}$. Besides, the effect of convection is ignored and the manufacture temperature is set $25^{\circ} \mathrm{C}$. In Fig. $1, \mathrm{AB}$ is defined as path 1 , and $\mathrm{BC}$ is defined as path 2 .

According to the flat model of particle, this model can be simplified as two-dimensional axisymmetric model by taking half of the longitudinal section and setting symmetry constraint boundary conditions on the symmetry plane in the analysis process. The solving of thermal stress is done by using indirect coupling method which uses Plane55 unit to complete temperature field calculation firstly and then convert Plane55 to Plane42 unit to calculate thermal stress.

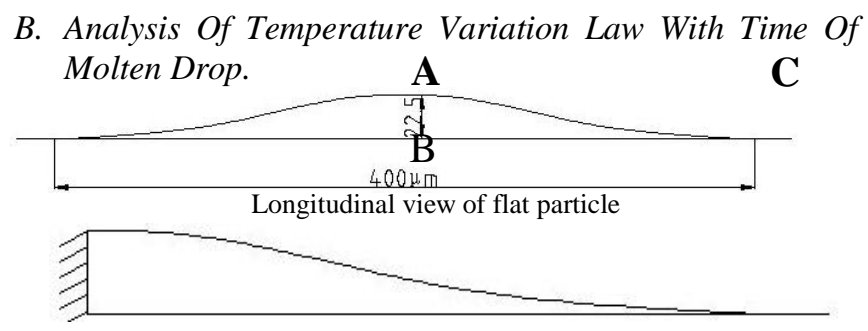

Figure 1. Two-dimensional axisymmetric model

The temperature contour map of molten drop in $100 \mu \mathrm{s}$ is shown in Fig. 2. The temperature variation curves of Points A and B in flat model is shown in Fig. 3. Fig. 2 shows that: The temperature of molten drop decreases from the center to the edge with the temperature in the center being the highest. Fig. 3 shows that: The temperature of Point A at the top of drop decreases gradually with time. The temperature of Point $B$ on the junction area increases firstly and then decreases. Finally, the temperature of Points A and B gradually drops to the same temperature. In $100 \mu \mathrm{s}$, the temperature of the whole drop trends to be $200^{\circ} \mathrm{C}$.

\section{Analysis of Stress Field Variation Law with Time of Molten Drop.}

The changes of radial and axial stress along path 1 are shown in Fig. 4. As it can be seen, after solidification of drop, along path $\mathrm{AB}$, the radial stress is tensile stress, and the axial stress is compressive stress. The radial stress changes more apparently than the axial stress, and both decrease with the axial size increasing. The maximum stress occurs at Point B, and such a large tensile stress may cause the coating to warp.

The variation laws of radial and axial stress along path 2 are shown in Fig. 5. It can be seen that: The radial stress of drop at the junction area with base is tensile stress which increases with the radial dimension increasing. The axial stress in the central area of drop is compressive stress which decreases with the radial size increasing, and then the compressive stress turns to tensile stress which alternates with compressive stress. Stress at the edge of the drop changes sharply, and the axial tensile stress at Point $\mathrm{C}$ gets to peak. Such a large tensile stress may lead to the initiation of micro-cracks at the edge of interface and expansion to the central area, resulting in the failure of coating layering. As to the axial stress $\sigma_{y}$, it has small compressive stress in the central area and small tensile stress at the edge. The axial stress in the central area is very little, almost zero.
Comparison shows that the axial stress is one magnitude smaller than the radial stress, the axial stress can be ignored and the radial stress can be only considered at the study of stress at the bonding interface.
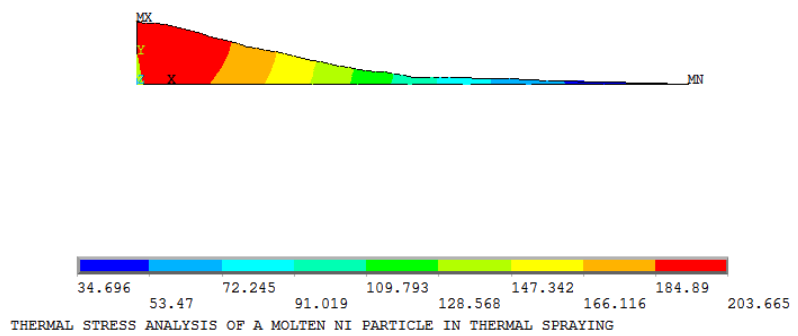

Figure 2. Temperature contour map of drop in $100 \mu \mathrm{s}$

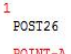

NNSYS

POINT-A
POIIT-

11:00:57

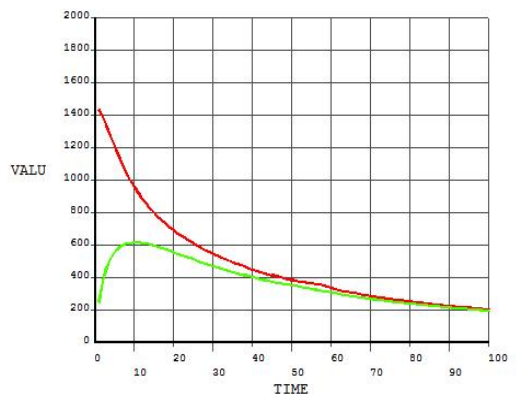

THERMAL STRESS ANALYSIS OF A MOLTEN NI PARTICLE IN THERMAL SPRAYING

Figure 3. Temperature variation curves of Points A and B

The three-dimensional radial and axial stress distribution in $100 \mu$ s after solidification of $1 / 4$ molten drop is shown in Fig. 6. As it can be seen, the stress of drop is in whole larger than the base with a relatively large stress abruptness. As the thermal expansion coefficient of nickel-based superalloy is higher than the base metal, the residual tensile stress occurs in the base after spraying; while due to the constraint of the base, residual compressive stress occurs in the coating whose maximum equivalent stress is near the base side in the drop. And as the shear stress is smaller, so it can be sure that radial stress is the main factor leading to residual thermal stress during the thermal spraying process.

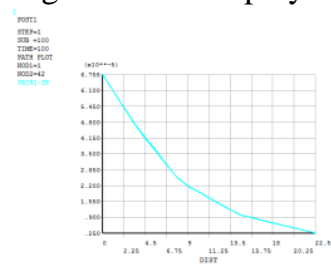

Radial direction
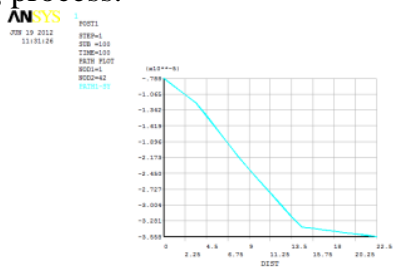

Axial direction
Figure 4. Stress curve along path 1 

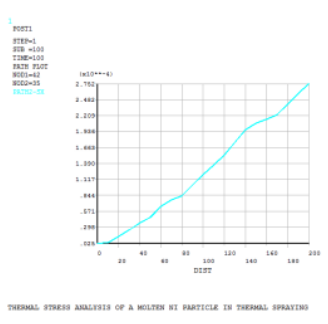

Radial direction
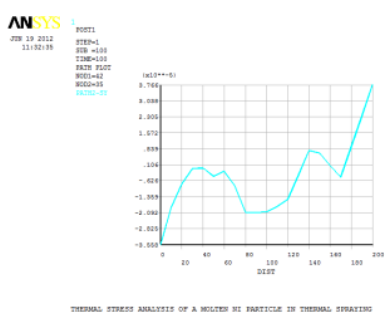

Axial direction

Figure 5. Stress curve along path 2

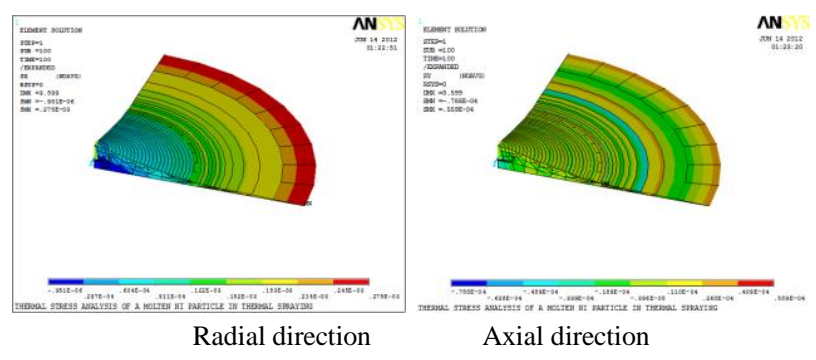

Figure 6. Three-dimensional stress distribution of 1/4 drop
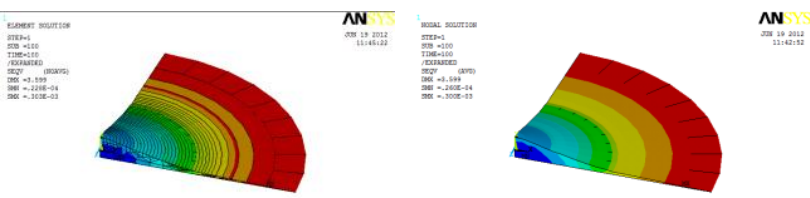

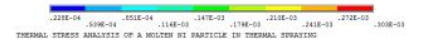

(a) (b)

Figure 7. Residual stress distribution (a) and residual strain distribution (b)

Fig. 7 shows precisely the total residual stress and strain distribution after deposition and solidification of drop in $100 \mu \mathrm{s}$. It can be seen that: There is large residual stress and strain at the edge of the drop, which may cause cracks, peeling or warping at the coating edge. Therefore, it is important for plasma spraying processing to control the temperature and optimize the processing parameters of coating. Fig. 8 shows the strain energy distribution of molten drop in $100 \mu \mathrm{s}$, and it can be seen that the total trend of strain energy of drop is that: From the central region of coating to the edge, because of the large difference of thermal expansion coefficient between nickel-based alloy and base, the region of strain energy enlarges with slight violation.

\section{Process Simulation Of Ni Particles Spraying On The Carbon Steel Base.}

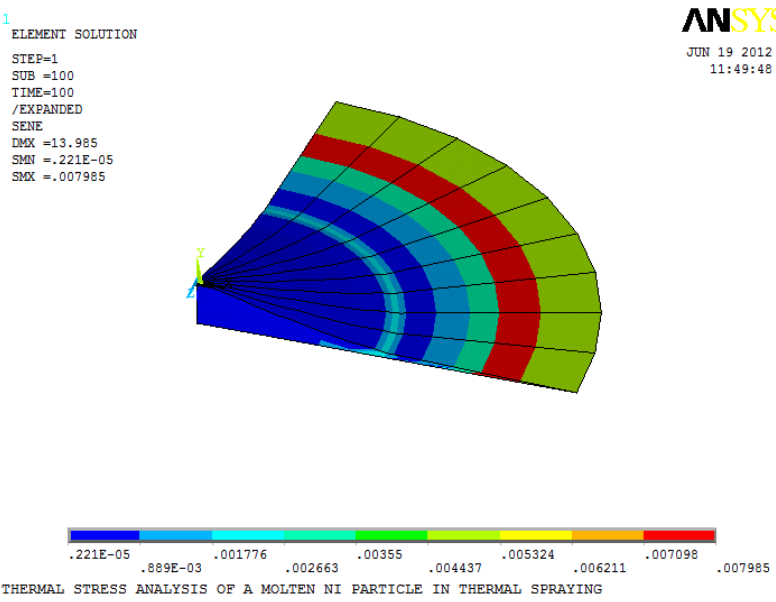

Figure 8. Strain energy distribution of drop in $100 \mu \mathrm{s}$ ELEMENTS

NN

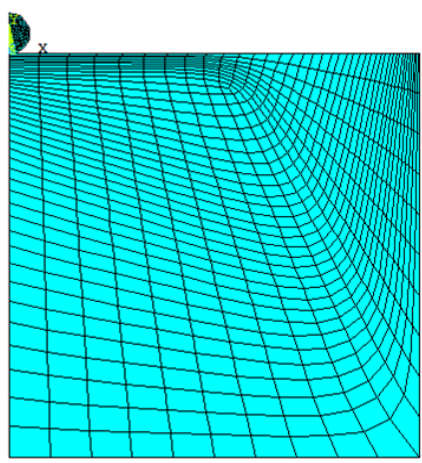

JoN 152012

high velcocity impact of spraying

Figure 9. FE model of particles colliding the base

In order to simulate better the collision and deposition process of $\mathrm{Ni}$ particles on carbon steel base with the stress and strain change during the process, and the process of deformation, ANSYS LS-DYNA module is adopted for modeling and simulation. Since the process of collision among $\mathrm{Ni}$ particles and base is symmetric, the physical model can be built as two-dimensional axisymmetric model. Taking computing time into account, the model size of base which cannot be too large is just 10 times the diameter size of particle, which causes no difficulty in observing the deformation area. The mathematical model of particles hitting the base is shown in Fig. 9. The stress and strain variation law after the collision are respectively shown in Fig. 10 and Fig. 11. The variation curve with time of the total energy during the process of collision and deposition of the particle is shown in Fig. 12. 


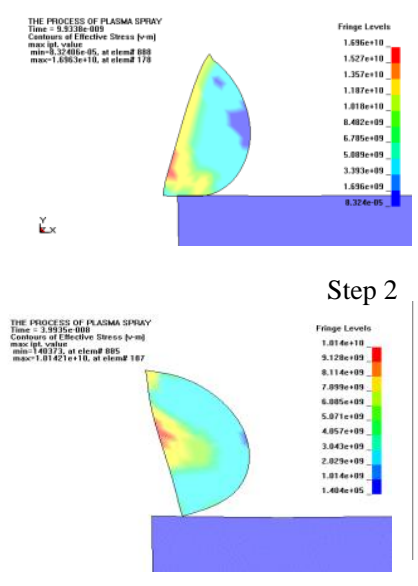

Step 6

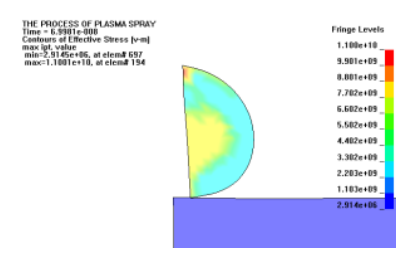

Step 10

Step 8

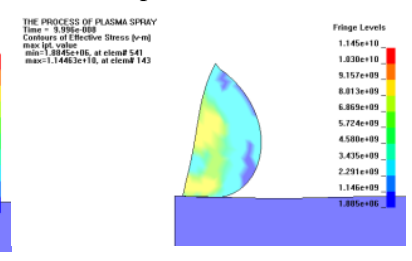

Step 12

Figure 10. Stress distribution in different steps in collision process

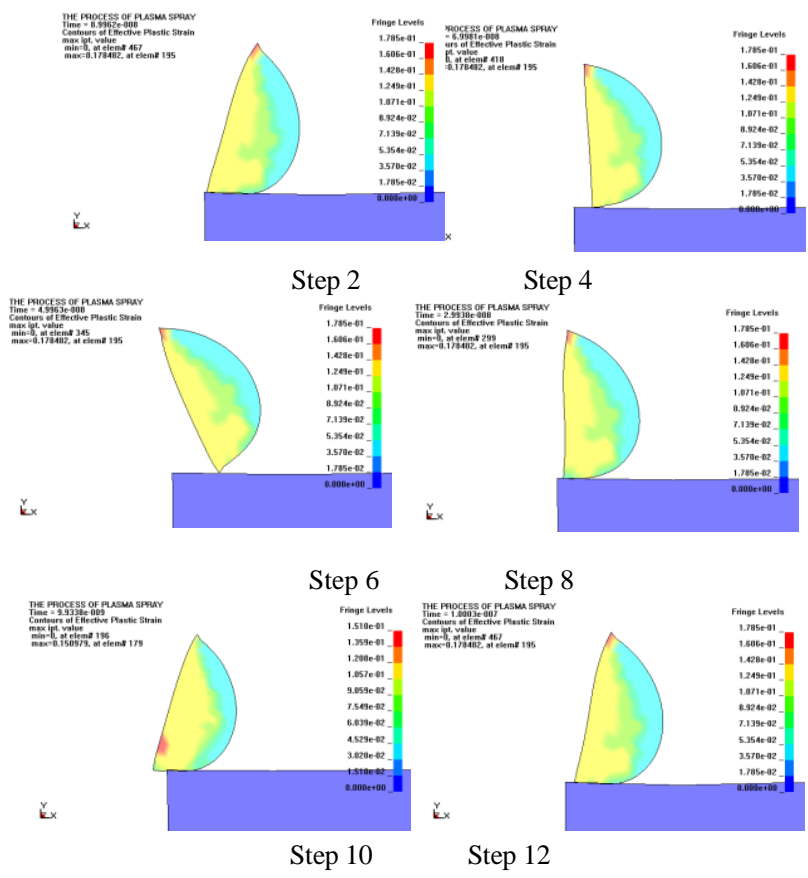

Figure 11. Stress distribution in different steps in collision process

The stress change process in Fig. 10 shows that as the Ni particles hit the carbon steel base, the stress in contact area among Ni particles and base first increases to maximum and then decreases gradually with particles having the trend to be flat. Due to the sputtering behavior of particles during the process of collision, the stress at the top of particle is the largest when the collision comes to an end. The plastic strain

change process in Fig. 11 shows that as the Ni particles hit the carbon steel base, the strain away from the contact area gradually increases to maximum while in contact area the strain gradually extends. It is suggested that the particles first hit on the base and deform gradually, and then they are attached to the base which causes the strain affected by the stress of Ni particles increases firstly and then decreases but the plastic strain of base expands gradually and does not change finally.

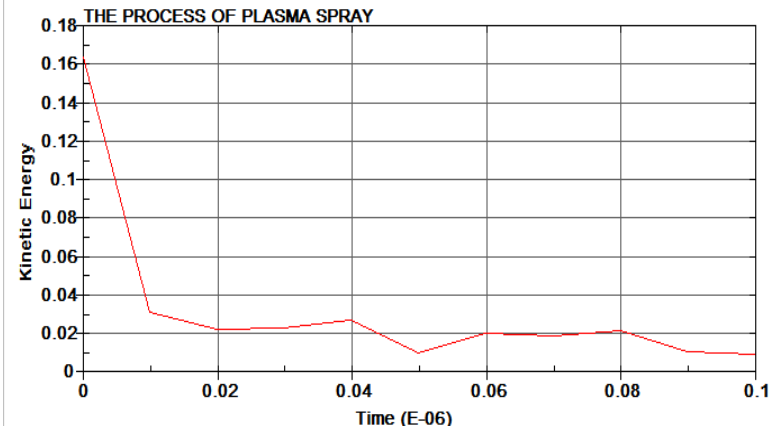

Figure 12. Curve of kinetic energy

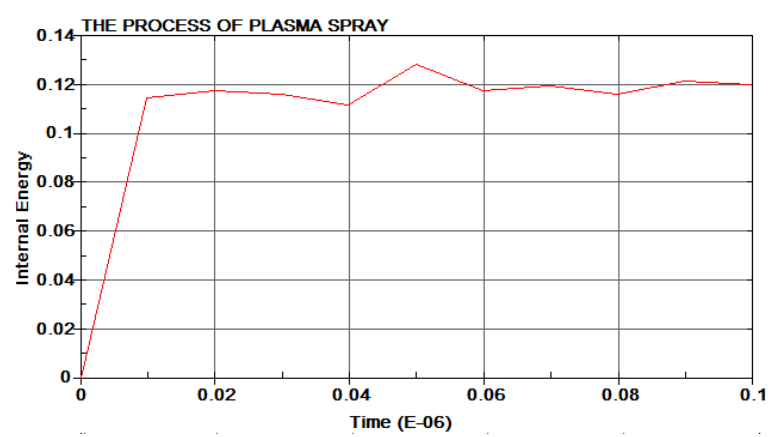

Figure 13. Curve of internal energy

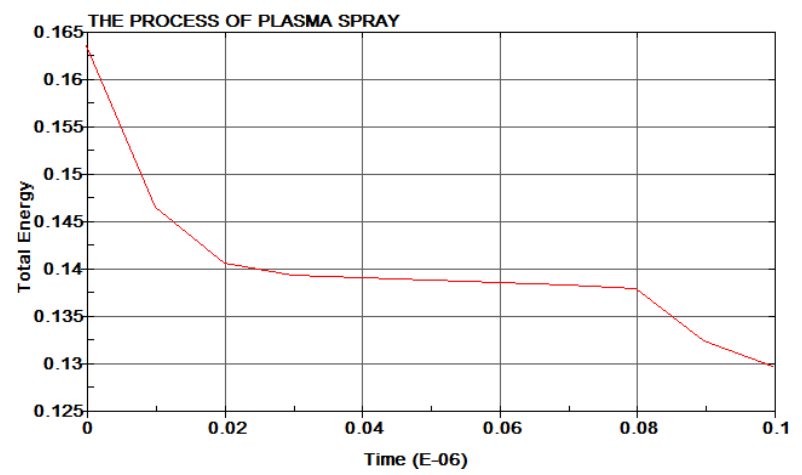

Figure 14. Curve of total energy

From the plastic strain figure, it can be seen apparently that the overall shape of particle has a certain relationship with the increasing spraying speed. There is strong plastic strain in the narrow contact area among particles and base with maximum strain concentrating around contact area and the surface of base has certain mesh deformation. From 
reference [4-7], it can be seen that with different spraying velocity, the mesh size have a great effect on the flat ratio of particle. The overall rule is that: The flat ratio decreases with the mesh size decreasing, and as the spraying velocity is near or greater than $500 \mathrm{~m} / \mathrm{s}$, metal sputtering phenomenon occurs around particles and base. The results in reference [4-7] have a certain difference with the simulation results of this paper in which the flat ratio of particle increases with spraying velocity.

Fig. 12, Fig. 13 and Fig. 14 separately shows the variation with time of kinetic energy, variation with time of internal energy and variation with time of total energy. It can be seen from the above figures that the kinetic energy decreases sharply at the beginning of collision and becomes stable and then gradually decreased when the maximum deformation is gotten. At the same time, the internal energy stabilizes after rapid increasing at the beginning, and the total energy is gradually reducing. The results show that during the collision process, plastic deformation occurs in both the particles and the base, which leads to the consumption of kinetic energy of particles. Most of the kinetic energy of particles and base is converted into internal energy. After the collision is complete, the kinetic energy of particles transfers into particles and base.

\section{CONCLUSIONS}

Through the above analyses and simulations, the following conclusions can be reached:

1. The temperature of flat particle decreases gradually along the center to edge, and the gradient of temperature in central area is higher. The temperature in junction area first increases, and then decreases with time, and become the same finally.

2. The radial stress in contact area among molten drop and carbon steel base is compressive stress in the central area and tensile stress at the edge, and tensile stress transforms rapidly to compressive stress with the radial size increasing. The axial stress in the central area is small compressive stress. The axial stress is one magnitude smaller than radial stress, and the stress in junction area is mainly radial stress.

3. The various preheating temperature of the base metal can change the distribution of stress in the interface, but cannot change the shape of stress distribution. With the preheating temperature increasing, the compressive stress in central area increases apparently, the tensile stress at the edge decreases clearly and the bonding strength among coating and base is enhanced. In this paper, only the heat exchanges among coating and base and the stress in coating with the same final temperature are analyzed. In fact, during the actual spraying process, the coating and base have convection with the external environment and finally cool to room temperature together after they have the same temperature. So the above analysis of residual stress provides foundation for further studying the stress among coating and base at room temperature.

4. The main form of residual stress that remained in the drop and base during the plasma spraying process is radial stress. Since the thermal expansion coefficient of drop is higher than base, the residual tensile stress occurs in the base while the residual compressive stress occurs in the coating after spraying. As residual stress has an important effect on the quality and performance of the coating, so properly controlling the residual stress distributions can effectively improve toughness and bonding strength of the interface and thereby enhance the life of workpiece.

\section{ACKNOWLEDGMENT}

The research work is supported by the Fundamental Research Funds for the Central Universities (3142013099).

\section{REFERENCES}

[1] Nylen P, Wigren J, Pejryd, Ansson M O. the modeling of coating thickness, heat transfer, and fluid flow and its correlation with the thermal barrier coating microstructure for a plasma sprayed gas turbine application. Journal of thermal Spray technology, 1999, 8(3):393-398.

[2] Nishiyama, Kuzuhara M, Solonenko O P, Kamiyama S. Numerical modeling of an impinging dusted plasma jet controlled by a magnetic field in a low pressure. Thermal Spray: Meeting the Challenges of the 21st Century. France: ASM International, 1998: 451-456.

[3] Bertangnoli M, Marchese M, Jacucci G. Modeling of Particles Impacting on a Rigid Sub-state under Plasma Spraying Conditions. J Thermal Spray Technology, Vol.1,1995:41-49.

[4] LI Wen-ya, LIAO Han-lin, LI Chang-jiu, et al. Numerical simulation of deformation behavior of $\mathrm{Al}$ particles impacting on $\mathrm{Al}$ substate and effect of surface oxide films on interfacial bonding in cold spraying. Applied Suface Science, 2007, 253(11):5084-5091.

[5] Assadi H, Gartner F,Stoltenhoff $\mathrm{T}$, et al. Bonding mechanism between particle substrate in cold gas spraying. Acta Materialia, 2003, 51(15): 4379-4394.

[6] Y. P. Wan ,V. Prasad ,G. X. Wang, et al. Model and Powder Particle heating, Melting, Resolidification, and Evaporation in Plas2ma Spraying Processes . Journal of Heat Transfer. 1999 ,121:691-699.

[7] M. Bussmann.S. Chandra. J. Mostaghimi. Numerical Results of off 2 Angle Thermal Spray particles Impact. Proceedings of UTSC 99. $1999.783-86$ 